\title{
Posicionamiento en buscadores de las webs oficiales de capitales de provincia españolas
}

\author{
Por Cristòfol Rovira, José Fernández-Cavia, Rafael Pedraza-Jiménez y Assumpció Huertas
}

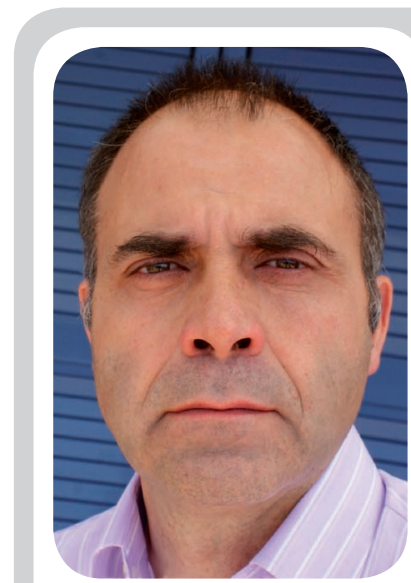

Cristòfol Rovira es profesor agregado del área de Biblioteconomía y Documentación de la Univ. Pompeu Fabra. Imparte docencia en las titulaciones de Publicidad y relaciones públicas, Comunicación audiovisual, así como en el Máster online en documentación digital, Máster en buscadores y en el Máster interuniversitario UB/UPF sobre Gestión de contenidos digitales. Investiga sobre herramientas para la evaluación automática de sedes web (DigiDocSpider). Forma parte del grupo DigiDoc del Depto. de Comunicación de la UPF.



José Fernández-Cavia es profesor titular del Departamento de Comunicación de la Universitat Pompeu Fabra y subdirector de investigación y postgrado en el mismo. Investigador principal del proyecto Nuevas estrategias de publicidad y promoción de marcas turísticas españolas en la Web, del Ministerio de Ciencia e Innovación. Sus líneas de investigación principales están relacionadas con la publicidad, las relaciones públicas y la comunicación turística.

http://www.marcasturisticas. org



Rafael Pedraza-Jiménez es profesor de la Univ. Pompeu Fabra y miembro del grupo de investigación DigiDoc de esta misma universidad. Imparte docencia en los estudios de Periodismo y Comunicación Audiovisual, así como en el Master interuniversitario en gestión de contenidos digitales (UB-UPF), el Master en buscadores (IDEC/UPF) y el Master online en documentación digital (IDEC/UPF). Participa en varios proyectos de investigación sobre la web semántica, ingeniería de ontologías, recuperación de información y técnicas de SEO/SEM.

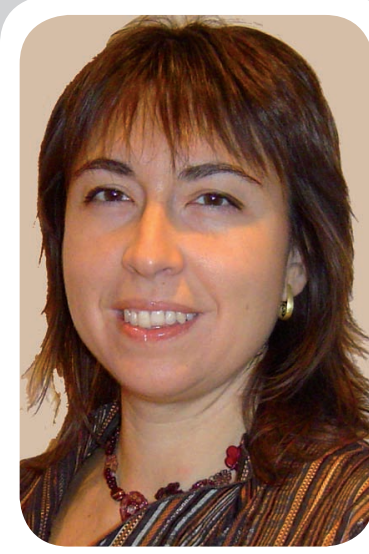

Assumpció Huertas es lic. en publicidad y relaciones públicas por la Univ. Autònoma de Barcelona y lic. en ciencias políticas y sociología por la misma universidad. Es doctora por la Univ. Rovira i Virgili y profesora agregada en la misma, donde imparte las asignaturas de teoría y técnica de las relaciones públicas (RRPP) y publicidad y RRPP en el ámbito local. También es profesora colaboradora en la UOC, donde imparte publicidad y RRPP I y profesora de redacción de mensajes en RRPP en la Escuela Superior de RRPP de Barcelona.

Resumen: Se estudia el posicionamiento en Google y Yahoo de las sedes web de las grandes corporaciones municipales españolas en búsquedas con la marca turística de la ciudad. Se analiza si se están promocionando de forma adecuada estas marcas en internet. Se presentan resultados del seguimiento diario del posicionamiento en Google y Yahoo durante 2009 de las búsquedas por las marcas turísticas de las capitales de provincia españolas. Los resultados indican que un $66 \%$ de las sedes web oficiales están posicionadas después del número 40 y que en el $46 \%$ de las búsquedas hay una sede web oficial entre las 10 primeras posiciones. Se han detectado evidencias empíricas sobre el peso de los enlaces entrantes y de las palabras clave para las estrategias de posicionamiento en buscadores.

Palabras clave: Posicionamiento, SEO, Marcas de ciudad, Webs municipales, Google, Yahoo.

Title: Search engine positioning of Spanish official websites of provincial capitals on searches of city brand

Abstract: Study of ranking in Google and Yahoo of big Spanish municipal corporation websites while searching by city tourist brand. Analysis of whether city tourist brands are promoting properly in the internet. Results of the daily ranking monitoring in Google and Yahoo are shown, for the searches made in 2009 by touristic brands of Spanish provincial capitals. The results show that $66 \%$ of official websites are situated after number 40 and, also, that in $46 \%$ of the searches made by touristic brands of province capital there is an official website within the top 10 positions. Empirical evidence about the weight of backlinks and keywords for SEO has also been detected.

Keywords: SEO, Place brand websites, Web site city hall, Google, Yahoo.

Rovira, Cristòfol; Fernández-Cavia, José; Pedraza-Jiménez, Rafael; Huertas, Assumpció. "Posicionamiento en buscadores de las webs oficiales de capitales de provincia españolas". El profesional de la información, 2010, mayo-junio, v. 19 , n. 3, pp. 277-284.

DOI: 10.3145/epi.2010.may.08 


\section{Introducción}

La importancia de internet es cada vez mayor para la comunicación de las marcas comerciales en general (McMillan, 2004; Ko; Cho; Roberts, 2005) y, en especial, para las llamadas marcas de lugar (place brands) (Morgan; Pritchard; Pride, 2004; Blain; Levy; Brent-Ritchie, 2005; Buhalis; Costa, 2006).

De la misma manera que no se entiende una marca comercial importante sin una representación adecuada en la Red, tampoco se entiende que una marca turística de territorio, sea ciudad, país o región, no utilice buena parte de sus recursos de comunicación en ese canal. Según la Organización Mundial de Turismo: "la información online es ahora la influencia principal en las decisiones del consumidor en los mercados más importantes" (World Tourism Organization; European Travel Commission, 2008).

Algunos autores consideran que se puede hablar de un nuevo tipo de turista, relacionado con los cambios de hábitos producidos por internet. Es una persona poco interesada en los paquetes turísticos tradicionales, menos habituada a esperas o retrasos, más exigente y sofisticada y, sobre todo, está acostumbrada a dirigirse directamente a los proveedores y a buscar por su cuenta información y adquirir billetes de avión o reservar hoteles desde la Web, en vez de confiar en las agencias de viajes tradicionales u otros intermediarios (Buhalis; Law, 2008).

Una de las formas más eficaces de presencia y comunicación en la Red es la creación y mantenimiento de un sitio web, tanto para las marcas turísticas como para las comerciales. A éstas les permiten proporcionar gran cantidad de información de forma ordenada, recuperable, personalizada y adaptada culturalmente (Tsikriktsis, 2002). Además crean experiencias virtuales de producto (Klein, 2003), construyen imagen de marca (Hollis, 2005) y pueden plantearse como un medio adecuado para la promoción de ventas, el marketing directo, la publicidad y las relaciones públicas (Cho; Cheon, 2005).

Los sitios web constituyen herramientas fundamentales de comunicación para las marcas turísticas (Palmer, 2005) y en particular, de las marcas de ciudad (Díaz-Luque, 2009). Con ellas se informa pero también se persuade, se convence, se ayuda a la toma de decisiones y finalmente se permite realizar reservas y compras. En el mercado de los destinos turísticos, los sitios web son un instrumento de mejora de la competitividad. El sitio web oficial de un destino puede resultar de importancia clave antes, durante y después del viaje. Puede ser de máxima utilidad a los viajeros en el momento de exploración y selección del destino, en la etapa de planificación del viaje, a la hora de reservar servicios, en el transcurso de la visita y en la fase posterior -el momento de comentarlo, compartir experiencias y realizar recomendaciones a otras personas- (World Tourism Organization; European Travel Commission, 2008). Pero ¿cómo debe ser un sitio web de una marca turística de lugar para que cumpla adecuadamente su función, para que no pase desapercibido en la world wide web y pueda atraer el máximo número de visitas?

Un elemento fundamental son las estrategias de posicionamiento, pues de nada sirve construir un sitio web oficial atractivo y eficaz desde el punto de vista de la comunicación y la comercialización del destino turístico, si después, cuando el potencial turista realiza su búsqueda en la Red, es incapaz de encontrarlo o, tal vez peor, termina navegando por una página no oficial sin ser consciente de ello.

Por esa razón el posicionamiento en buscadores (search engine optimization, SEO) es una disciplina básica en cualquier campaña de marketing en internet. Un volumen altísimo del tráfico de visitas que recibe una web proviene de los buscadores (algunos autores señalan porcentajes superiores al 80\%). Por tanto, será de suma importancia para cualquier web sobre marcas estar en las primeras posiciones en los resultados de búsquedas con palabras clave que coincidan con la marca promocionada.

\section{Factores críticos de posicionamiento}

Para evitar fraudes, los buscadores no informan de manera concreta y detallada acerca de los factores que intervienen en el algoritmo de ordenación de sus resultados. No obstante, es sabido que el posicionamiento se basa en factores internos y externos (Casares, 2008; Clay; Esparza, 2008; Enge et al., 2009; Maciá; Gosende, 2009; SEOmoz, 2009). Entre los factores internos, todos los autores coinciden en resaltar la presencia de las palabras clave en determinadas partes del contenido de la página o de la cabecera, estructura hipertextual de la sede web, antigüedad, territorialidad del dominio... Entre los externos, los más decisivos son el número de enlaces recibidos y la presencia de una parte de estos enlaces en sedes web con autoridad o popularidad.

El estudio SEOmoz (2009) va un poco más allá y en base a la opinión de 72 expertos internacionales en posicionamiento otorga un peso a cada uno de los factores que han sido considerados como determinantes (figura 1).

\section{Objetivos y método}

Este trabajo de investigación se plantea determinar si las grandes corporaciones municipales españolas están promocionando de forma adecuada sus marcas turísticas de ciudad en internet. Para ello se ha analizado 




Figura 1. Factores que intervienen en el posicionamiento de Google. Fuente: SEOmoz (2009)

http://www.seomoz.org/article/search-ranking-factors

el posicionamiento en Google y Yahoo durante 2009 de las sedes web creadas por sus ayuntamientos.

Presentaremos los resultados de un año de seguimiento diario de los dominios mejor posicionados con palabras clave que son marcas turísticas de las ciudades españolas capitales de provincia. Entre estas primeras posiciones se identifican y analizan los dominios pertenecientes a las corporaciones municipales para elaborar un ranking de las que han promocionando de forma más efectiva su marca turística en la Red durante 2009.

Por otro lado, la investigación ha sido planteada de forma exploratoria para obtener evidencias empíricas sobre los siguientes aspectos:

- Cuáles son los factores de éxito que hacen posible un buen posicionamiento, sobre todo en relación con los enlaces entrantes.

- Hasta qué punto los resultados de Google y Yahoo son coincidentes en posicionamiento y en número de resultados.

Las conclusiones de este análisis permiten ofrecer recomendaciones prácticas basadas en evidencias empíricas para mejorar el posicionamiento de las webs oficiales de turismo de las ciudades españolas. Estas conclusiones podrían extrapolarse a otras webs de características similares.

\section{Hipótesis de trabajo}

Se han asumido los siguientes presupuestos que actúan como hipótesis de trabajo y que han guiado el diseño de nuestra investigación:

Hipótesis 1: de acuerdo con Casares (2008), Clay y Esparza (2008), Enge et al., (2009), Maciá y Gosende (2009) y en especial SEOmoz (2009), hay 3 factores con un peso importante en el PageRank (Google) y en el WebRank (Yahoo) y que por tanto están influyendo de manera decisiva en el posicionamiento de una página web:

- Presencia de las palabras clave en la etiqueta $t i$ tle
- Presencia de las palabras clave en el nombre del dominio

- Elevado número de enlaces recibidos desde páginas que a su vez reciben una elevada cantidad de enlaces.

Hipótesis 2: las páginas bien posicionadas en Google también lo serán en Yahoo, sobre todo cuando se analiza un período largo de tiempo y se consideran sólo las primeras posiciones.

\section{Obtención de datos}

La cibermetría ha elaborado procesos y herramientas para la obtención de datos sobre las sedes web. A menudo se usan robots, spiders o mapeadores elaborados específicamente para recoger datos útiles para los indicadores cibermétricos, como por ejemplo Astra site manager, SocSciBot, Microsoft site analyst, WebCount, Web trends, Хетu link sleuth... (Arroyo-Vázquez, 2004). No obstante, se trata de herramientas complejas de personalizar y útiles sólo para un determinado tipo de indicadores. Es preferible usar los datos que proporcionan los principales motores de búsqueda (Aguillo, 2009; Aguillo; Granadino, 2006) intentando evitar los errores o sesgos que a menudo cometen estos instrumentos. La obtención de datos de los buscadores generalistas es la práctica más común de los grupos de investigación en cibermetría. Este enfoque parece ser también el más indicado para la recogida de datos para el posicionamiento.

La investigación se ha llevado a cabo utilizando DigiDocSpider (Pedraza-Jiménez; Codina; Rovira, 2008), un programa informático de tipo rastreador elaborado por el grupo de investigación DigiDoc del Departamento de Comunicación de la Universitat Pompeu Fabra. Las capacidades del rastreador incluyen la revisión de sitios web disponibles en internet extrayendo y analizando los elementos del código fuente previamente seleccionados. DigiDocSpider puede remitir el url analizado a servicios abiertos accesibles en línea para después compilar los resultados obtenidos e incorporarlos a sus resultados. Esta prestación se utiliza para aplicar de forma automática validadores del tipo xhtml, accesibilidad, etc., y también para obtener datos de los buscadores, como por ejemplo, el número de enlaces recibidos o la posición de un url en determinadas búsquedas.

En total, DigiDocSpider puede recopilar de forma automática más de cien indicadores relativos a cinco aspectos básicos en la evaluación de sitios web: accesibilidad, metadatos, posicionamiento de los buscadores, visibilidad y calidad del código xhtml.

En esta investigación hemos aplicado este programa para obtener datos de Google y Yahoo de forma no 
intrusiva ni saturante. Hemos obtenido diariamente los dominios situados entre las primeras cuarenta primeras posiciones en las búsquedas para los nombres de las ciudades capitales de provincia españolas (marcas de ciudad) en todos los idiomas oficiales.

Las búsquedas han sido realizadas en las versiones internacionales de los buscadores y con las palabras clave del nombre de ciudad sin aplicar ninguna de las opciones avanzadas de los buscadores, como búsqueda por frases o restricciones por título. De este modo imitamos el comportamiento de un usuario no experto que corresponde al público objetivo de la oferta turística de las grandes ciudades españolas.

\section{Resultados. Ranking de dominios mejor posicionados}

En las tablas 1 y 2 se presentan los tres dominios mejor posicionados en Google y Yahoo con las palabras clave de las marcas de ciudad españolas que son capitales de provincia. Por razones de espacio sólo listamos las 12 primeras marcas por orden alfabético. Son resultados integrados de los valores obtenidos durante los 365 días del año 2009 y con un volumen de 11.000 datos diarios procesados. Las webs oficiales pertenecientes a ayuntamientos están marcadas con un asterisco.

Los resultados muestran que existe un porcentaje relativamente elevado de grandes sedes web generalistas como Google news, Flickr, YouTube y en especial Wikipedia, que aparecen entre las tres posiciones en el $100 \%$ de los casos en Google y en el 93,4\% en Yahoo.

Otro aspecto que muestran los resultados es la baja presencia de webs oficiales entre estas tres primeras posiciones de búsqueda. Aparecen en un $21 \%$ de las búsquedas en Google y tan solo en un 5\% en Yahoo. Este dato parece indicar que las campañas de posicionamiento van dirigidas específicamente a Google. No obstante, la presencia de sedes web oficiales entre las primeras posiciones aumenta hasta el $46 \%$ si analizamos hasta la posición número diez, como veremos en el siguiente apartado.

\section{"Los responsables municipales deberían prestar atención a la descripción de su ciudad en Wikipedia, que aparece siempre en las primeras posiciones en las búsquedas"}

\section{Ranking de dominios oficiales}

Posteriormente se han analizado los mejores dominios de las webs oficiales de las capitales de provincia españolas. En la tabla 3 se muestra el ranking de las mejor posicionadas. Son datos de la mediana de la posición en Google durante el año 2009 con búsquedas por palabras clave del nombre o marca de la ciudad. La toma de datos se ha realizado diariamente teniendo en cuenta las 40 primeras posiciones.

"El $66 \%$ de las webs oficiales de capitales de provincia españolas son 'invisibles' en Google ya que están más allá de la posición $40 "$

\begin{tabular}{|c|c|c|c|c|c|}
\hline \multicolumn{2}{|r|}{ A Coruña } & \multicolumn{2}{|r|}{ Alacant } & \multicolumn{2}{|r|}{ Albacete } \\
\hline 1 & http://en.wikipedia.org & 1 & http://en.wikipedia.org & 1 & http://en.wikipedia.org \\
\hline 2 & *http://www.coruna.es & 2 & *http://www.alicante-ayto.es & 2 & *http://www.albacete.com \\
\hline 3 & http://www.galiciaguide.com & 3 & http://www.urbanrail.net & 3 & http://www.albacetebalompie.com \\
\hline \multicolumn{2}{|r|}{ Alicante } & \multicolumn{2}{|r|}{ Almería } & \multicolumn{2}{|r|}{ Ávila } \\
\hline 1 & http://www.alicante-spain.com & 1 & http://en.wikipedia.org & 1 & http://en.wikipedia.org \\
\hline 2 & http://www.alicante.com & 2 & http://www.andalucia.com & 2 & http://www.spain.info \\
\hline 3 & http://en.wikipedia.org & 3 & http://www.wunderground.com & 3 & http://www.cyberspain.com \\
\hline \multicolumn{2}{|r|}{ Badajoz } & \multicolumn{2}{|r|}{ Barcelona } & \multicolumn{2}{|r|}{ Bilbao } \\
\hline 1 & http://en.wikipedia.org & 1 & http://en.wikipedia.org & 1 & http://en.wikipedia.org \\
\hline 2 & http://www.euroresidentes.com & 2 & http://www.fcbarcelona.com & 2 & http://www.spain.info \\
\hline 3 & http://www.wfrmuseum.org.uk & 3 & http://www.barcelona.com & 3 & http://www. virtourist.com \\
\hline \multicolumn{2}{|r|}{ Bilbo } & \multicolumn{2}{|r|}{ Burgos } & \multicolumn{2}{|r|}{ Cáceres } \\
\hline 1 & http://www.bilbos.com & 1 & http://en.wikipedia.org & 1 & http://en.wikipedia.org \\
\hline 2 & http://en.wikipedia.org & 2 & http://www.red2000.com & 2 & *http://www.ayto-caceres.es \\
\hline 3 & http://www.bilbobaggins.net & 3 & *http://www.turismoburgos.org & 3 & http://whc.unesco.org \\
\hline
\end{tabular}

Tabla 1. Dominios mejor posicionados en Google durante 2009 con las palabras clave de las marcas turísticas de las capitales de provincia españolas (sólo 12 primeras alfabéticamente) http://www.marcasturisticas.org 


\begin{tabular}{|c|c|c|c|c|c|}
\hline \multicolumn{2}{|r|}{ A Coruña } & \multicolumn{2}{|r|}{ Alacant } & \multicolumn{2}{|r|}{ Albacete } \\
\hline 1 & http://en.wikipedia.org & 1 & http://en.wikipedia.org & 1 & http://en.wikipedia.org \\
\hline 2 & http://gospain.about.com & 2 & http://alacant.indymedia.org & 2 & http://commons.wikimedia.org \\
\hline 3 & http://www.encyclopedia.com & 3 & http://www.premiervillasinthesun.com & 3 & http://www.wunderground.com \\
\hline \multicolumn{2}{|r|}{ Alicante } & \multicolumn{2}{|r|}{ Almería } & \multicolumn{2}{|r|}{ Ávila } \\
\hline 1 & http://en.wikipedia.org & 1 & http://en.wikipedia.org & 1 & http://en.wikipedia.org \\
\hline 2 & http://www.alicante-spain.com & 2 & http://www.andalucia.com & 2 & http://www.kayak.com \\
\hline 3 & http://wikitravel.org & 3 & http://www. virtualtourist.com & 3 & http://www.avila.edu \\
\hline \multicolumn{2}{|r|}{ Badajoz } & \multicolumn{2}{|r|}{ Barcelona } & \multicolumn{2}{|r|}{ Bilbao } \\
\hline 1 & http://en.wikipedia.org & 1 & http://en.wikipedia.org & 1 & http://en.wikipedia.org \\
\hline 2 & http://www.euroresidentes.com & 2 & http://www.barcelona.com & 2 & http://wikitravel.org \\
\hline 3 & http://www.expedia.com & 3 & http://www.aboutbarcelona.com & 3 & http://www. virtourist.com \\
\hline \multicolumn{2}{|r|}{ Bilbo } & \multicolumn{2}{|r|}{ Burgos } & \multicolumn{2}{|r|}{ Cáceres } \\
\hline 1 & http://www.bilbo.com & 1 & http://en.wikipedia.org & 1 & http://www.encyclopedia.com \\
\hline 2 & http://en.wikipedia.org & 2 & http://www.red2000.com & 2 & http://www.facebook.com \\
\hline 3 & http://www.tuckborough.net & 3 & http://www.wunderground.com & 3 & http://www.flickr.com \\
\hline
\end{tabular}

Tabla 2. Dominios mejor posicionados en Yahoo durante 2009 con las palabras clave de las marcas turísticas de las capitales de provincia españolas (sólo 12 primeras alfabéticamente) http://www.marcasturisticas.org

El 66\% de webs oficiales de capitales de provincia españolas son invisibles cuando son buscadas en Google por el nombre de la ciudad, ya que están más allá de la posición 40. Los datos todavía son más desalentadores

\begin{tabular}{|c|c|c|}
\hline $\begin{array}{l}\text { Palabra } \\
\text { clave }\end{array}$ & Dominio & $\begin{array}{c}\text { Mediana } \\
\text { del orden } \\
\text { en Google }\end{array}$ \\
\hline Gasteiz & http://www.vitoria-gasteiz.org & 2 \\
\hline Huesca & http://www.huescaturismo.com & 2 \\
\hline A Coruña & http://www.coruna.es & 2 \\
\hline Albacete & http://www.albacete.com & 2 \\
\hline Lleida & http://www.Ileidatur.com & 2 \\
\hline Iruña & http://www.pamplona.net & 3 \\
\hline Logroño & http://www.logroturismo.org & 3 \\
\hline Ávila & http://www.avilaturismo.com & 3 \\
\hline Ourense & http://www.turismourense.com & 3 \\
\hline Pamplona & http://www.pamplona.net & 3 \\
\hline Ceuta & http://www.ceuta.es & 3 \\
\hline Zaragoza & http://www.zaragoza.es & 4 \\
\hline Málaga & http://www.malagaturismo.com & 4 \\
\hline $\begin{array}{l}\text { La } \\
\text { Coruña }\end{array}$ & http://www.coruna.es & 4 \\
\hline León & http://www.leon.es & 4 \\
\hline Cádiz & http://www.cadizturismo.com & 4 \\
\hline A Coruña & http://www.turismocoruna.com & 4 \\
\hline Burgos & http://www.turismoburgos.org & 4 \\
\hline Huelva & http://www.ayuntamientohuelva.es & 5 \\
\hline Melilla & http://www.melillaturismo.com & 5 \\
\hline $\begin{array}{l}\text { Las } \\
\text { Palmas } \\
\text { de Gran } \\
\text { Canaria }\end{array}$ & http://www.grancanaria.com & 5 \\
\hline
\end{tabular}

Tabla 3. Ranking de posicionamiento de las mejores sedes web oficiales de marcas turísticas de capitales de provincia española en Yahoo, donde tan sólo un 8,5\% está presente antes de la posición 40. El 100\% de las situadas entre las 40 primeras posiciones en Yahoo aparecen también en estas posiciones en Google, en cambio tan solo un $25 \%$ de las localizadas en Google también están presentes en Yahoo. La presencia en las primeras posiciones de Yahoo es muy reducida, un $92 \%$ de las sedes web oficiales analizadas quedan después de la posición 40 .

No obstante hay dos datos muy positivos. Un $84 \%$ de las marcas de ciudad analizadas posee la sede web oficial entre las 40 primeras posiciones en Google.

En Google, en el $46 \%$ de las búsquedas por marcas turísticas de capital de provincia hay alguna sede web oficial entre las 10 primeras posiciones.

Cabe destacar que la importancia política o económica de las ciudades no queda reflejada en el ranking de posicionamiento. Ciudades como Huesca, Lleida o Albacete quedan mucho mejor situadas que Madrid o Barcelona.

\section{"En el $46 \%$ de las búsquedas por marcas turísticas de capital de provincia en Google, hay alguna sede web oficial entre las 10 primeras posiciones"}

\section{Evidencias empíricas sobre las hipótesis}

La finalidad de esta investigación es obtener algunas evidencias que permitan confirmar la veracidad del 
planteamiento inicial. Una de las hipótesis se basa en que el posicionamiento depende en gran parte de los enlaces recibidos y de la presencia de las palabras clave en la etiqueta title y en el nombre del dominio.

Más del $90 \%$ de las páginas iniciales de las sedes web oficiales analizadas tienen en la etiqueta title y en el nombre del dominio web las palabras clave correspondientes al nombre de la ciudad. Tan solo en los casos con nombres de ciudad en dos lenguas oficiales, se opta por una de ellas quedando la segunda denominación fuera de estos lugares de privilegio. Por tanto el diseño de las sedes web de las grandes corporaciones municipales es correcto en este punto, aunque no siempre este acierto se traduce en un buen posicionamiento, como se ha dicho. Por otra parte, también cabe subrayar que el $62 \%$ de los dominios que se encuentran entre las 3 primeras posiciones no contienen las palabras clave.

Como consecuencia, deducimos que la presencia de las palabras clave en la etiqueta title y en el dominio no son tan importantes, sino que cuentan como factores complementarios. Tienen un peso reducido en los algoritmos de posicionamiento y por tanto su importancia es menor de la que habíamos previsto al iniciar este estudio. Esta conclusión concuerda con la bibliografía reciente sobre posicionamiento. La mayoría de los expertos del informe SEOmoz (2009) otorgan a estos dos factores una importancia elevada o muy elevada dentro del apartado de "palabras clave en la página". Sin embargo, este apartado tiene tan sólo un peso del 15\% en el global de todos los factores (figura 1).

Por otro lado, con los datos que proporcionan los propios buscadores es muy complicado obtener evidencias empíricas sobre cómo actúan los enlaces recibidos en el posicionamiento. Yahoo permite hacer búsquedas sobre enlaces recibidos externos a una determinada url. En cambio Google sólo ofrece datos de enlaces recibidos, sin poder diferenciar entre los externos y los que provienen de la propia web receptora.

Los enlaces útiles para el posicionamiento son los externos aunque no todos tienen el mismo peso. La importancia de cada enlace depende del valor del PageRank (Google) y del WebRank (Yahoo) de las páginas emisoras del enlace. A su vez estos parámetros dependen de los enlaces recibidos estableciéndose un procedimiento recursivo de cálculo que sintetiza en un solo valor, y en escala logarítmica ${ }^{1}$, la cantidad y la calidad de los enlaces recibidos. La complejidad del cálculo del PageRank ha crecido con el tiempo a medida que Google intenta evitar páginas que han sido posicionadas de forma fraudulenta. Es por ello que Google últimamente está hablando también de TrustRank. A pesar de estas complejidades y la escala logarítmica, cabría esperar que a mayor PageRank mejor posición, en especial cuando son constantes al menos dos factores im- portantes relacionados con las palabras clave. Este fue nuestro planteamiento inicial, tal y como se indicaba en la primera hipótesis.

Los resultados muestran que el PageRank de las páginas iniciales de las sedes web oficiales analizadas está situado entre 0 y 7 . Tan sólo 6 sedes web tienen $\mathrm{Pa}$ geRank de 7 y todas ellas ocupan en Google posiciones entre las 10 primeras. Por otro lado, hay 22 sedes web con PageRank de 6 y todas, excepto 3, también ocupan posiciones entre las 20 primeras.

En relación al PageRank no hemos detectado resultados incongruentes $\mathrm{y}$, tal como indica la teoría, en igualdad de otras condiciones, cuanto mayor es el $\mathrm{Pa}$ geRank, mejor posicionamiento. Dicho de otra forma, hemos obtenido evidencias empíricas que indican que cuanto más y mejores son los enlaces entrantes, mejor será el posicionamiento, tal como habíamos indicado en uno de los apartados de la primera hipótesis.

\section{"Las palabras clave en la etiqueta title y en el dominio tienen un peso reducido en los algoritmos de posicionamiento"}

\section{Coincidencias y discrepancias entre Google y Yahoo}

Hay una extensa bibliografía sobre los errores, inconsistencias y sesgos en los resultados de los buscadores, tanto en relación a su cobertura temporal (Bar-Ilan, 2005), como a la cobertura de dominios (Snyder; Rosenbaum, 1999), los números de resultados (Notess, 2000; Rousseau, 1999; BarIlan, 2000; 2002) o la profundidad de la indexación (Thelwall, 2000). Los problemas son múltiples y mal documentados en las ayudas de los buscadores. Desde una perspectiva comercial y en relación con la calidad del servicio, no son deficiencias graves, pero en un contexto de investigación académica, los errores deben ser identificados y minimizados, en la medida de lo posible. La comparación de los resultados de Google y Yahoo puede aportar valiosas evidencias sobre el grado de consistencia de los datos numéricos de los listados de ambos motores de búsqueda.

\footnotetext{
"En igualdad de otras condiciones, cuanto mayor es el PageRank mejor posicionamiento"
} 
Es evidente que Google y Yahoo son distintos desde la perspectiva del posicionamiento, sus índices son distintos y sus algoritmos de posicionamiento también. No obstante, cabría esperar cierta coincidencia cuando se observan los primeros resultados de un análisis de posicionamiento con un volumen grande de datos. Nuestra hipótesis es que los dominios que de forma constante quedan entre las primeras posiciones en un buscador también tendrían que quedar bien posicionados en el otro.

Para obtener evidencias sobre esta hipótesis hemos realizado dos grupos de dominios, los situados entre las 10 primeras posiciones y los que aparecen entre la posición 11 y la 40. A continuación hemos calculado el coeficiente de correlación entre las variables posición en Google y posición en Yahoo en ambos grupos obteniendo resultados muy elocuentes. Las correlaciones en los grupos de las 10 primeras posiciones son moderadas y significativas, en cambio en los grupos a partir de la posición 10 se obtienen correlaciones muy bajas e incluso no significativas.

\begin{tabular}{|l|c|}
\hline \multicolumn{1}{|c|}{ Grupo de dominios } & $\begin{array}{c}\text { Coeficiente de } \\
\text { correlación rho de } \\
\text { Spearman }\end{array}$ \\
\hline Posición <10 en Google & 0,547 \\
\hline Posición 11 a 40 en Google & 0,239 \\
\hline
\end{tabular}

Tabla 4. Coeficiente de correlación entre las posiciones de Google y Yahoo. Selección de grupos en función de Google

\begin{tabular}{|l|c|}
\hline \multicolumn{1}{|c|}{ Grupo de dominios } & $\begin{array}{c}\text { Coeficiente de } \\
\text { correlación rho de } \\
\text { Spearman }\end{array}$ \\
\hline Posición <10 en Yahoo & 0,518 \\
\hline Posición 11 a 40 en Yahoo & $-0,004$ \\
\hline
\end{tabular}

Tabla 5. Coeficiente de correlación entre las posiciones de Google y Yahoo. Selección de grupos en función de Yahoo

Resultaría muy arriesgado extrapolar estas conclusiones y decir que en general todos los resultados de Yahoo y Google son coincidentes cuando se analizan a largo plazo las 10 primeras posiciones. No obstante, la contundencia de estos resultados es una clara evidencia sobre la segunda hipótesis planteada.

El análisis de coincidencia se ha realizado con los dominios que se encuentran entre las 40 primeras posiciones en ambos buscadores. Pero tan sólo un $46 \%$ de los dominios que aparecen en Google están también en Yahoo. En cambio, todos los que aparecen en Yahoo están también en Google. Estos datos no son estadísticamente representativos y por tanto las conclusiones son sólo evidencias parciales. No obstante, todo parece indicar que los índices de Google son más completos y que las discrepancias entre Google y Yahoo estarían más en la cobertura de la Red que en la ordenación de los resultados.

\section{"Las coincidencias en posicionamiento entre Google y Yahoo son significativas sólo en las 10 primeras posiciones"}

\section{Conclusiones}

En esta investigación hemos detectado algunos datos desalentadores sobre el posicionamiento de las sedes webs oficiales de los ayuntamientos de capitales de provincia españolas. Un 66\% son invisibles cuando son buscadas en Google por el nombre de la ciudad, ya que aparecen más allá de la posición 40. En Yahoo los datos son todavía peores, tan solo un $8,5 \%$ de las webs oficiales se encuentran antes de la posición 40. No obstante, también hay motivos para el optimismo ya que en un $84 \%$ de las marcas de ciudad analizadas aparece alguna sede web oficial entre las 40 primeras posiciones en Google.

Las webs estudiadas están bien diseñadas para el posicionamiento en relación con la presencia de la marca turística en la etiqueta title o en el nombre del dominio. No obstante, todo parece indicar que la cantidad y la calidad de enlaces entrantes son mejorables. Los responsables de la imagen corporativa municipal deberán tener especial atención en la mejora de los enlaces recibidos cuando diseñen campañas de posicionamiento. También sería aconsejable hacer campañas específicas para Yahoo y revisar la página de la ciudad en Wikipedia ya que aparece siempre entre las 3 primeras posiciones.

En el transcurso de la investigación se han obtenido algunas evidencias sobre el peso de los enlaces entrantes para el posicionamiento y del alto grado de coincidencia en la ordenación de resultados de Google y Yahoo cuando se analizan las primeras posiciones.

En futuros trabajos se deberán confirmar los indicios detectados y profundizar en algunos de los aspectos todavía no analizados, como por ejemplo si existen diferencias significativas en el posicionamiento de las marcas de ciudad en función del idioma del nombre de la ciudad, en los casos con varias denominaciones.

\section{Nota}

1. Explicado de forma simple, que el PageRank $(P R)$ sea logarítmico significa que si para pasar de $P R=1$ a $P R=2$ se necesita recibir n enlaces, para pasar de $P R=2$ a $P R=3$ se necesitan 10n enlaces, y para alcanzar un $P R=4$ se necesitan 100n, etc. 


\section{Financiación}

Esta investigación ha sido financiada parcialmente por el proyecto "Nuevas estrategias de publicidad y promoción de las marcas turísticas españolas en la web" (CSO 2008-02627), del Ministerio de Ciencia e Innovación (España). Puede encontrarse más información en:

http://www.marcasturisticas.org

\section{Bibliografía}

Aguillo, Isidro F. (coord.). Factor de impacto y visibilidad de 4.000 sedes web universitarias españolas. Proyecto estudios y análisis 2004. Ea20040020. Equipo del Laboratorio de Internet - Centro de Información y Documentación Científica (Cindoc-Csic). Madrid, 16 de junio de 2005. Versión 2.0

http://univ.micinn.fecyt.es/univ/html/informes/estudios_analisis/ resultados_2004/ea0020/EA2004-0020.pdf

Aguillo, Isidro F. "Problemas técnicos, metodológicos y documentales en la elaboración de rankings basados en indicadores web". XI Jornadas españolas de documentación. Fesabid, 2009.

http://www.fesabid.org/zaragoza2009/actas-fesabid-2009/15-27.pdf

Aguillo, Isidro F.; Granadino, Begoña. "Indicadores web para medir la presencia de las universidades en la Red". Revista de universidad y sociedad del conocimiento, 2006, v. 3 , n. 1 .

http://www.uoc.edu/rusc/3/1/dt/esp/aguillo_granadino.pdf

Arroyo-Vázquez, Natalia. Métodos y herramientas para la extracción de datos en cibermetría: el software académico y comercial. Tesis de licenciatura de la Univ. de Salamanca, Depto. de Biblioteconomía y Documentación, y del Laboratorio de Internet del Centro de Información y Documentación Científica (Cindoc, hoy Iedcyt), 2004.

http://hdl.handle.net/10261/4497

Bar-Ilan, Judit. "Evaluating the stability of the search tools HotBot and Snap: a case study". Online information review, 2000, v. 24, n. 6, pp. 439 449.

Bar-Ilan, Judit. "How much information search engines disclose on the links to a web page? A longitudinal case study of the 'cybermetrics' home page". Journal of information science, 2002, v. 28, n. 6, pp. 455-466.

Bar-Ilan, Judit. "Expectations versus reality - Search engine features needed for Web research at mid 2005". Cybermetrics, 2005, v. 9. http://www.cindoc.csic.es/cybermetrics/articles/v9i1p2.html

Blain, Carmen; Levy, Stuart E.; Brent-Ritchie, J. R. "Destination branding: insights and practices from destination management organizations". Journal of travel research, 2005, v. 43, n. 4, pp. 328-338.

Buhalis, Dimitrios; Costa, Carlos. Tourism, management dynamics. Trends, management and tools. Oxford: Elsevier Butterworth Heinemann, 2006

Buhalis, Dimitrios; Law, Rob. "Progress in information technology and tourism management: 20 years on and 10 years after the internet - The state of eTourism research". Tourism management, 2008, v. 29, n. 4, pp. 609-623.

Casares, Javier. Guía de referencia SEO. Barcelona: OJO Internet, SL, 2008.

http://www.manualdeposicionamiento.com/guia-de-referencia-seo/

Cho, Chang-Hoan; Cheon, Hongsik-John. "Cross-cultural comparisons of interactivity on corporate web sites: the United States, the United Kingdom, Japan and South Korea". Journal of advertising, 2005, v. 34, n. 2, pp. 99-115.

Clay, Bruce; Esparza, Susan. Search engine optimization all-in-one for dummies. Hoboken: Wiley, 2009.

Díaz-Luque, Pablo. "Official tourism web sites and city marketing". En: Gascó-Hernández, Mila; Torres-Coronas, Teresa. Information communication technologies and city marketing. Digital opportunities for cities around the world. Hershey: Information Science Reference, 2009.

Enge, Eric; Spencer, Stephan; Fishkin, Rand; Stricchiola, Jessie. The art of SEO: mastering search engine optimization. Sebastopol, CA: O'Reilly, 2009.

Hollis, Nigel. "Ten years of learning on how online advertising builds brands". Journal of advertising research, 2005, June, v. 45, pp. 255-268.

Klein, Lisa R. "Creating virtual product experiences: the role of telepresence". Journal of interactive marketing, 2003, v. 17, n. 1, pp. 41-55.

Ko, Hanjun; Cho, Chang-Hoan; Roberts, Marilyn S. "Internet uses and gratifications. A structural equation model of interactive advertising". Journal of advertising, 2005, v. 34, n. 2, pp. 57-70.

Maciá, Fernando; Gosende, Javier. Posicionamiento en buscadores. Edición 2009. Barcelona: Anaya Multimedia, 2009.

McMillan, Sally J. “Internet advertising: one face or many?” En: Schumann, David W.; Thorson, Esther. Internet advertising: theory and research, Psychology Press, 2004.

Morgan, Nigel; Pritchard, Annette; Pride, Roger. Destination branding: creating the unique destination proposition. Oxford: Elsevier, 2004.

Notess, Greg. "Search engine inconsistencies". Online, March 2000. http:// www.onlinemag.net/OL2000/net3.html

Palmer, Adrian. "The internet challenge for destination marketing organizations". En: Morgan, Nigel; Pritchard, Annette; Pride, Roger. Destination branding: creating the unique destination proposition. Oxford: Elsevier, 2004.

Pedraza-Jiménez, Rafael; Codina, Lluís; Rovira, Cristòfol. "Semantic web adoption: online tools for web evaluation and metadata extraction". The 8th Intl Flins conf on computational intelligence in decision and control, Madrid, Sept. 21-24, 2008

Rousseau, Ronald. "Daily time series of common single word searches in AltaVista and NorthernLight". Cybermetrics, 1999, v. 2/3, n. 1, paper 2. http://www.cindoc.csic.es/cybermetrics/articles/v2i1p2.html

Rovira, Cristòfol. "Anàlisi i avaluació dels llocs web de televisions en català". BiD: textos universitaris de biblioteconomia i documentació, 2007, n. 18.

http://www.ub.edu/bid/18rovir3.htm

Rovira, Cristòfol; Marcos, Mari-Carmen. "Metadatos en revistas-e de documentación de libre acceso". El profesional de la información, 2006, v. 15, n. 2, pp. 136-144.

http://eprints.rclis.org/9452/1/doc.1.pdf

SEOmoz. Advanced SEO tactics: On beyond keyword research (GF401). http://www.seomoz.org/article/search-ranking-factors

Snyder, Herbert; Rosenbaum, Howard. "Can search engines be used as tools for web-link analysis? A critical view". Journal of documentation, 1999, v. 55, n. 4, pp. 375-384.

Tsikriktsis, Nikos. "Does culture influence website quality expectations? An empirical study". Journal of service research, 2002, v. 5, n. 2, pp. 101112 .

Thelwall, Mike. "Web impact factors and search engine coverage". Journal of documentation, 2000, v. 56, n. 2, pp. 185-189.

http://wlv.openrepository.com/wlv/handle/2436/3141

World Tourism Organization; European Travel Commission. Handbook on e-marketing for tourism destinations. Madrid, 2008.

http://pub.world-tourism.org/WebRoot/Store/Shops/Infoshop/48EA/148E/ 19AC/BB41/5091/C0A8/0164/579A/080926_handbook_e-marketing_ excerpt.pdf

\section{Cristòfol Rovira, José Fernández-Cavia, Rafael Pedra- za-Jiménez. Universitat Pompeu Fabra, Barcelona.} cristofol.rovira@upf.edu jose.fernandez@upf.edu rafael.pedraza@upf.edu

Assumpció Huertas. Universitat Rovira Virgili, Tarragona.

sunsi.huertas@urv.cat 